\title{
Screening for Methicillin-resistant Staphylococcus aureus Carriage on the Hands of Healthcare Workers: An Assessment for Hand Hygiene Practices
}

\author{
Anuradha Sharma ${ }^{1}$, Jitu M Kalita², Vijaya L Nag ${ }^{3}$
}

\begin{abstract}
Background: Methicillin-resistant Staphylococcus aureus (MRSA) is capable of causing a wide range of infections. Colonized healthcare workers (HCWs) and contaminated hand-touch surfaces act as a potential source of MRSA in hospitals. This study was conducted to detect the carriage of MRSA in the hands of HCWs during patient care to check awareness among HCWs to follow proper hand hygiene protocol.

Materials and methods: This study was a cross-sectional point prevalence study done in wards and intensive care units (ICUs) of a tertiary care hospital. Hand cultures were collected from HCWs after the clinical rounds, without prior information about the procedure. It was done on three consecutive days to include maximum HCWs from the hospital. Cultures were taken before and after the use of alcohol-based hand rub. Hand cultures were obtained by asking HCWs to touch the surface of chromogenic screening agar for MRSA with their fingertips and thumbs of both the hands.

Results: Of a total of 62 HCWs screened, 32 (51.61\%) were positive for MRSA. Among these, seven were doctors. After using alcohol-based hand rub, six HCWs were still positive for MRSA. Another important finding on this screening agar was detection of Candida on the hands of HCWs. Conclusion: Regular monitoring of hand hygiene compliance is vital to prevent the spread of nosocomial infections. The MRSA screening agar is rapid, simple, cost-effective, and useful to identify the carriage of not only MRSA but also Candida (in the wake of nosocomial outbreaks with Candida auris) in the hands of HCWs. Further studies are required to evaluate the transmission rate of MRSA from HCWs to patients in Indian hospitals.

Keywords: Chromogenic agar, Hand culture, Hand hygiene, Methicillin-resistant Staphylococcus aureus carriage.

Indian Journal of Critical Care Medicine (2019): 10.5005/jp-journals-10071-23296
\end{abstract}

\section{INTRODUCTION}

Nosocomial infections contribute to the important cause of morbidity and mortality in any hospital setting. ${ }^{1}$ Contact among healthcare workers (HCWs) and patients appears to be the most common route of transmission of methicillin-resistant Staphylococcus aureus (MRSA). ${ }^{2}$ Methicillin-resistant S. aureus is capable of causing a wide range of infections. ${ }^{3}$ It is believed that $\mathrm{HCW}$ s are more likely to be colonized than persons in the general population because of increased exposure. ${ }^{4}$ They increase the risk of spreading MRSA and other organisms from person to person with direct hand contact during a single opportunity. Therefore, colonized HCWs and contaminated hand-touch surfaces act as a potential source of MRSA in hospitals. ${ }^{5}$

This study was conducted to detect the carriage of MRSA in the hands of HCWs during patient care to evaluate the effective practice of hand hygiene protocol. After celebrating the "Global Hand Washing Day" on October 15 and subsequently the "International Infection Prevention Week" from October 16 to 21, 2017, we wanted to assess the follow-up awareness among HCWs.

\section{Materials and Methods}

This study was a cross-sectional point prevalence study of three consecutive days and was conducted among HCWs posted in all wards and intensive care units (ICUs) of a tertiary care hospital. The limitation in this study is small sample size as the study setting involves a newly established hospital and the numbers of beds were 400 at the time of study. Many HCWs either used hand rub
${ }^{1-3}$ Department of Microbiology, All India Institute of Medical Sciences, Jodhpur, Rajasthan, India

Corresponding Author: Jitu M Kalita, Department of Microbiology, All India Institute of Medical Sciences, Jodhpur, Rajasthan, India, Phone: +91 7042653469, e-mail: kalita.jitumoni@gmail.com

How to cite this article: Sharma A, Kalita JM, Nag VL. Screening for Methicillin-resistant Staphylococcus aureus Carriage on the Hands of Healthcare Workers: An Assessment for Hand Hygiene Practices. Indian J Crit Care Med 2019;23(12):590-592.

Source of support: Nil

Conflict of interest: None

before giving hand cultures or avoided giving it and therefore were excluded from the study. The HCWs were asked not to the use hand rub, as because we needed real-time carriage rate; therefore, if they tried to use it seeing us, we excluded them from the study.

A total number of $62 \mathrm{HCWs}$ were included in this study; of which, 14 were doctors. Hand cultures were obtained from HCWs after the clinical rounds without prior information about the procedure. Cultures were taken before and after the use of alcohol-based hand rub (after 1 minute of use). Hand cultures were obtained by asking HCWs to touch the surface of screen agar (MeReSa Chromogenic Agar from HiMedia Laboratory Pvt. Ltd, Mumbai, India) with their fingertips and thumbs of both the hands. Culture plates were incubated at $35^{\circ} \mathrm{C}$ aerobically for 48 hours. Bluish green colonies were further processed by conventional methods (Gram's stain, catalase test, slide coagulase test, and tube coagulase test).

(-) The Author(s). 2019 Open Access This article is distributed under the terms of the Creative Commons Attribution 4.0 International License (https://creativecommons. org/licenses/by-nc/4.0/), which permits unrestricted use, distribution, and non-commercial reproduction in any medium, provided you give appropriate credit to the original author(s) and the source, provide a link to the Creative Commons license, and indicate if changes were made. The Creative Commons Public Domain Dedication waiver (http://creativecommons.org/publicdomain/zero/1.0/) applies to the data made available in this article, unless otherwise stated. 
Methicillin resistance was confirmed by using cefoxitin disk $(30 \mu \mathrm{g})$ as surrogate marker. Resistance against other antimicrobial agents was tested by Clinical and Laboratory Standards Institute (CLSI) 2017 guidelines: ciprofloxacin $(5 \mu \mathrm{g})$, clindamycin $(2 \mu \mathrm{g})$, cotrimoxazole/ trimethoprim-sulfamethoxazole (TMP/SMX, 1.25/23.75 $\mu \mathrm{g}$ ), erythromycin $(15 \mu \mathrm{g})$, and gentamicin $(10 \mu \mathrm{g})$, on Mueller-Hinton agar with 24 hours of incubation at $35^{\circ} \mathrm{C}$. For internal quality control a known clinical isolate of MRSA was used as a positive control and S. aureus ATCC 25923 as a negative control. Other colorless growth on the screening agar was identified as Candida spp.

\section{Statistical Analysis}

The interpretation and analysis of the data were done by using Microsoft Excel. The quantitative data were expressed as numbers and percentages.

\section{Results}

Of a total of 62 HCWs screened for MRSA, 32 (51.61\%) were positive. Among these 32 MRSA-positive HCWs, 7 (21.88\%) were doctors and 25 (78.12\%) were other HCWs including nursing staff. After the use of alcohol-based hand rub, 6 (9.68\%) HCWs were still positive for MRSA.

Antimicrobial susceptibility pattern of MRSA isolates are shown in Figure 1. We also retrospectively analyzed the prevalence of MRSA in our hospital. A total of 29 MRSA isolates were reported from 1,524 samples during the 3 months preceding this study. Of these 29 isolates, only 7 were from indoor patients and 3 from pediatric ward. In this ward, the isolation of MRSA from the hands of HCWs was around $70 \%$. Another interesting finding was that HCWs with long nails (3/5) showed the growth of Candida spp. on this screening agar for MRSA.

\section{Discussion}

On the world map, Asia has the highest prevalence rates of MRSA. It is the most populated region in the world and self-medication with over-the-counter antimicrobial agents is a norm to treat common infections. This high antibiotic selection pressure among the overcrowded inhabitants creates an environment that is suitable for the rapid development and efficient spread of numerous multidrugresistant pathogens. ${ }^{6}$ In a multi-centric Indian study (2008-2009),

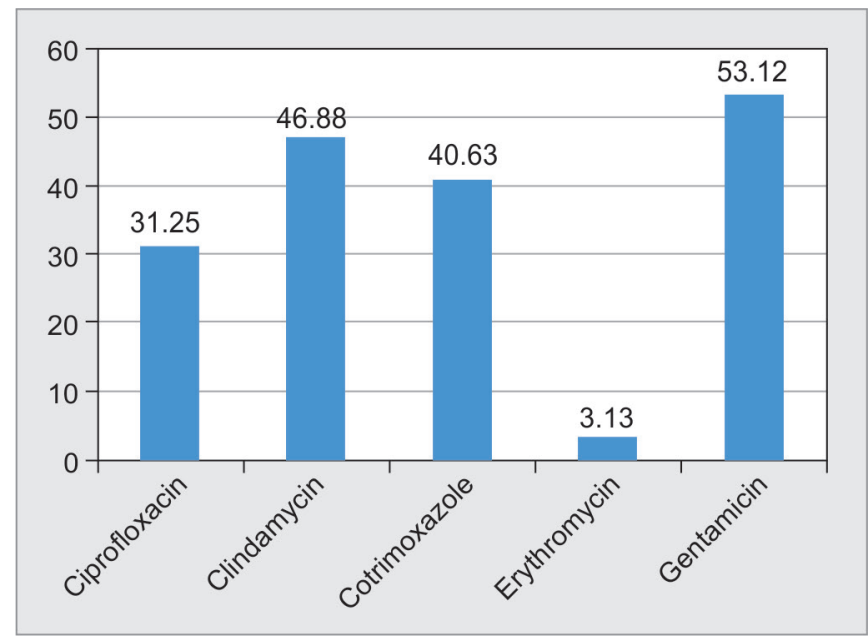

Fig. 1: Drugs sensitivity among methicillin-resistant Staphylococcus aureus isolates among patients the overall prevalence rate of MRSA was $41 \% .{ }^{7}$ Various Indian studies show MRSA nasal carriage rate of $1.8-6.6 \%$ among HCWs. ${ }^{8-10}$ In Nepal, the MRSA carriage rate was reported as $2 \% .{ }^{11} \mathrm{~A}$ very high MRSA nasal carriage rate of $38.9 \%$ is reported from Nigeria. ${ }^{12}$

Present study wanted to assess hand hygiene practices among our HCWs during real-life conditions with most convenient samples. It showed that $51.61 \%$ HCWs' hand cultures was positive for MRSA before using hand rub and after use $9.68 \%$ was still positive. We were trying to show the compliance of HCWs to hand hygiene and also demonstrate that if they use hand rub on the visibly dirty hands, it will not bring the desired outcome of removing MRSA. Those HCWs positive for MRSA after using alcohol-based hand rub may be because of they using the rub on their visibly dirty hands. The collection time between the use of hand rub and hand culture was 1 minute, which may not be sufficient. This could be another reason for culture positivity for MRSA after using hand rub. But we did not get any reference about adequate time to remove MRSA from hands after the use of hand rub. The hand rub was International Organization for Standardization certified and was used adequately by the staff. In another study, MRSA was detected in 39\% before hand hygiene and in $13 \%$ after hand hygiene, which is comparable to our study. ${ }^{13}$ Hospital environment has commonly touched surfaces. They can get contaminated either through HCWs' hands or via infected or colonized patients. Admission to a room previously occupied by a patient infected with MRSA, vancomycin-resistant Enterococcus species (VRE), or Clostridium difficile increases the risk of the subsequent patient to acquire the same pathogen. Wille et al. report that the use of inadequate concentrations of disinfectants can lead to persistence of MRSA in the hands of HCWs. In their study, MRSA was isolated from the mobile phones of HCWs. It is well known that HCWs compliance with hand hygiene is lower following environmental contact (50\%) compared to direct patient contact (80\%). The contributors to this practice are understaffing, high workload, insufficient knowledge, and may be more significantly underestimating the importance of cleaning and disinfection of hands. Hence, training and assessment is continuously needed regarding this simple infection prevention measure. ${ }^{14}$

Till recently, hospitals focused on the control of healthcareassociated MRSA (HA-MRSA) lineages, which are poorly adapted for persistence in the community. This situation has undergone a fundamental shift with the emergence of community-associated MRSA (CA-MRSA), which can colonize young and healthy population for long periods. Furthermore, CA-MRSA is now displacing previously dominant HA-MRSA lineages. ${ }^{15}$ The HCWs who are at the interface between the hospital and the community may serve as agents of cross transmission. ${ }^{4}$ The crowded living conditions with high antibiotic pressure are ideal to select and transmit MRSA strains not only in hospitals but also in community and in animal population (poultry). This will result in more use of vancomycin and subsequently lead to increased prevalence and spread of vancomycin-resistant S. aureus (VRSA) and VRE. Though at present sporadic reports are available in resource-poor countries, this constitutes a substantial public health threat, especially, where diagnostic facilities are largely lacking and appropriate therapy is frequently unaffordable. ${ }^{6}$ In the United States, patients are screened for MRSA carriage before admission to ICUs and kept in isolation till reports clear them. When colonized patients are not isolated, susceptible patients are at risk of acquiring MRSA at a rate of approximately $1 \%$ per day. Screening of MRSA relies on rapid screening test such as chromogenic agar or 
polymerase chain reaction. The treatment cost and unfavorable patient outcomes are more dangerous and expensive than the screening tests. ${ }^{16}$ Finally, the goal of any surveillance is to improve performance. Muller and Detsky have described two approaches for performance improvement in hand hygiene, namely, evidencebased and indicator-based strategies. Evidence-based studies are designed to improve patient outcomes and would ultimately in the long run improve staff adherence rates to the suggested practices. ${ }^{17}$ Every year on May 5 th, the World Health Organization and its committed partners all around the world celebrate the World Hand Hygiene Day as a call to action for health workers and also to stimulate the general public for why hand hygiene is so crucially important. This year, the slogan is "It's in your hands-prevent sepsis in health care."18

\section{ConcLusion}

Regular monitoring of hand hygiene compliance is vital to prevent the spread of nosocomial MRSA and thereby the emergence of VRE and VRSA. The MRSA screening agar is rapid, simple, cost-effective, and useful to identify the carriage of not only MRSA but also Candida (it is important in the wake of nosocomial outbreaks with highly adverse outcomes due to Candida auris). Further studies are required to evaluate the transmission rate of MRSA from HCWs to patients in Indian hospitals.

\section{References}

1. Ducel J, Fabry L, Nicolle G. Prevention of hospital acquired infections: a practical guide. WHO Rep 2002;2:1-3.

2. Schaberg DR, Culver DH, Gaynes RP. Major trends in the microbial etiology of nosocomial infection. Am J Med 1991;91(3B):72S-75S. DOI: 10.1016/0002-9343(91)90346-Y.

3. Oguzkaya-Artan M, Baykan Z, Artan C, Avsarogullari L. Prevalence and risk factors for methicillin resistant Staphylococcus aureus carriage among emergency department workers and bacterial contamination on touch surfaces in Erciyes University Hospital, Kayseri, Turkey. Afr Health Sci 2015;15(4):1289-1294. DOI: 10.4314/ahs.v15i4.31.

4. Radhakrishna M, D'Souza M, Kotigadde S, Vishwas-Saralaya K, Shashidar-Kotian M. Prevalence of methicillin resistant Staphylococcus aureus carriage amongst health care workers of critical care units in Kasturba Medical College Hospital, Mangaluru, India. J Clin Diagn Res 2013;7(12):2697-2700. DOI: 10.7860/JCDR/2013/5160.3735.

5. Kei J, Richards JR. The prevalence of methicillin-resistant Staphylococcus aureus on inanimate objects in an urban emergency department. J Emerg Med 2011;41(2):124-127. DOI: 10.1016/ j.jemermed.2008.08.002.
6. Chen CJ, Huang YC. New epidemiology of Staphylococcus aureus infection in Asia. Clin Microbiol Infect 2014;20(7):605-623. DOI: 10.1111/1469-0691.12705.

7. Joshi S, Ray P, Manchanda V, Bajaj J, Chitinis DS, Gautam V, et al. Methicillin resistant Staphylococcus aureus (MRSA) in India: prevalence \& susceptibility pattern. Indian J Med Res 2013;137(2): 363-369.

8. Mathanraj S, Sujatha S, Sivasangeetha K, Parija SC. Screening for methicillin-resistant Staphylococcus aureus carriers among patients and health care workers of a tertiary care hospital in south India. Indian J Med Microbiol 2009;27(1):62-64.

9. Goyal R, Das S, Mathur M. Colonisation of methicillin resisitant Staphylococcal aureus among health care workers in a tertiary care hospital of Delhi. Indian J Med Sci 2002;56(7):321-324.

10. Vinodhkumaradithyaa A, Uma A, Srinivasan M, Ananthalakshmi I, Nallasivam $P$, Thirumalaikolundusubramanian $P$, et al. Nasal carriage of methicillin-resistant Staphylococcus aureus among surgical unit staff. Jpn J Infect Dis 2009;62(3):228-229.

11. Shakya B, Shrestha S, Mitra T. Nasal carriage rate of methicillin resistant Staphylococcus aureus among at National Medical College Teaching Hospital, Birgunj, Nepal. Nepal Med Coll J 2010;12(1): 26-29.

12. Fadeyi A, Adeboye MAN, Fowotade A, Nwabuisi C, Bolaji BO, Oyedepo $\mathrm{OO}$, et al. Methicillin resistant Staphylococcus aureus carriage amongst healthcare workers of the critical care units in a Nigerian Hospital. Am J Infect Dis 2010;6(1):18-23.

13. Kocak Tufan Z, Irmak H, Bulut C, Cesur S, Kinikli S, Demiroz AP, et al. The effectiveness of hand hygiene products on MRSA colonization of health care workers by using CHROMagar MRSA. Mikrobiyol Bul 2012;46(2):236-246.

14. Wille I, Mayr A, Kreidl P, Bruhwasser C, Hinterberger G, Fritz A, et al. Cross-sectional point prevalence survey to study the environmental contamination of nosocomial pathogens in intensive care units under real-life conditions. J Hosp Infect 2018;98(1):90-95. DOI: 10.1016/ j.jhin.2017.09.019.

15. Harris SR, Cartwright Edward JP, Estee Torok M, Holden Matthew TG, Nicholas Brown M, Amanda Ogilvy-Stuart L, et al. Whole-genome sequencing for analysis of an outbreak of meticillin-resistant Staphylococcus aureus: a descriptive study. Lancet Infect Dis 2013;13(2):130-136. DOI: 10.1016/S1473-3099(12)70268-2.

16. Whittington MD, Curtis DJ, Atherly AJ, Bradley $C J$, Lindrooth RC, Campbell JD, et al. Screening test recommendations for methicillinresistant Staphylococcus aureus surveillance practices: a costminimization analysis. Am J Infect Control 2017;45(7):704-708. DOI: 10.1016/j.ajic.2016.12.014.

17. Larson E. Monitoring hand hygiene: meaningless, harmful, or helpful? Am J Infect Control 2013;41(5 Suppl):S42-S45. DOI: 10.1016/ j.ajic.2012.09.029.

18. It's in your hands-prevent sepsis in health care. WHO slogan on 5th May 2018. 